\title{
Attending to the Bodily Self
}

\begin{abstract}
Humphreys and Sui provide a powerful theoretical framework to explain processing biases towards self-related information. However, the framework is primarily applied to information relevant to a conceptual self-representation. Here, we show a similar processing bias for information related to a bodily self-representation, grounded in sensorimotor representations. Furthermore, we can use bodily illusions to explore the ways in which bodily selfassociations can affect our perceptual and attentional processing. It is possible to extend the current framework to take into account these effects, and we argue that this will yield considerable benefits for our understanding of self-relevance.
\end{abstract}

In the target article, Humphreys and Sui (henceforth H\&S) produce a novel framework to explain the effects of self-relevance on attention and perception. H\&S base much of their reasoning on findings of processing biases towards arbitrary shapes that have been briefly associated with the self. However, research on the self frequently distinguishes between two forms of self-representation: a 'conceptual' self, formed of a rich network of associative and semantic information, and a 'bodily' self, grounded in sensorimotor representations (Farmer \& Tsakiris, 2012). In this commentary we discuss the benefits of extending H\&S's framework, which currently focuses primarily on the conceptual self, to encompass the bodily self.

H\&S make three key points regarding the effects of self-relevance on attention. First, they discuss the distinct processing advantages shown towards self-relevant stimuli, such as one's own name. Second they demonstrate that arbitrary stimuli, such as shapes, can be incorporated in to the conceptual self through associative learning. Finally they show that processing advantages are extended to these newly self-relevant stimuli.

We argue that these points also apply to the bodily self. First, there is considerable evidence of processing bias for information related to the bodily self. For example, sensory integration is enhanced when perceptual stimuli are associated with our own bodies. This enhancement results in increased tactile sensitivity when a visual stimulus is concurrently viewed on our own body (Serino et al., 2008) and when auditory stimuli are perceived as close to our body (Ladavas, 2002). Attentional orienting is also altered by bodily self-relevance, with increased physiological stress response to the sight of painful stimuli approaching one's own body compared to another's body (Farmer et al., 2012).

Secondly, just like the conceptual self, the bodily self is malleable. Synchronous visuotactile stimulation elicits an embodied association over non-self-bodies (Botvinick \& Cohen, 1998; Tsakiris, 2008). This embodied association also occurs when tactile information is replaced with cardiac information, for example, when viewing a virtual body which is pulsing in synchrony with one's own heartbeat (Aspell et al., 2013).

Finally, once an embodied self-association has been established with another body, the processing advantages previously reserved for one's own body are extended to the other's 
body. For example, the enhanced integration of visuotactile (Aspell et al., 2013) and audiotactile (Maister et al., 2015a) information is now evident when viewing the other's body. Furthermore, threatening stimuli directed towards the other's body now elicits the same physiological stress responses as threats directed towards one's own body (Farmer et al., 2012).

We argue that neural regions involved in bodily self-representation should be included in H\&S's self-attention network. Brain areas such as the anterior insula and temporoparietal junction play a key role both in bodily (e.g. Tsakiris, 2010) and conceptual (Denny et al., 2012) self-representation, and these areas may support close bidirectional interactions between conceptual and bodily self-relevance (Maister et al., 2015b). Given the importance of embodied processing in social cognition (Farmer \& Tsakiris, 2012; Maister et al., 2015b), the inclusion of bodily self-processing in the model would extend its explanatory power further into the social domain, by providing a coherent, integrated account of self-relevance.

Aspell, J. E., Heydrich, L., Marillier, G., Lavanchy, T., Herbelin, B., \& Blanke, O. (2013). Turning body and self inside out: visualized heartbeats alter bodily self-consciousness and tactile perception. Psychological Science, 24(12), 2445-2453.

Botvinick, M., \& Cohen, J. (1998). Rubber hands "feel" touch that eyes see. Nature, 391, 756.

Critchley, H. D., Wiens, S., Rotshtein, P., Ohman, A., \& Dolan, R. J. (2004). Neural systems supporting interoceptive awareness. Nature Neuroscience, 7(2), 189-195.

Denny, B. T., et al., (2012). A meta-analysis of functional neuroimaging studies of self-and other judgments reveals a spatial gradient for mentalizing in medial prefrontal cortex. Journal of Cognitive Neuroscience, 24(8), 1742-1752.

Farmer, H., Tajadura-Jiménez, A., \& Tsakiris, M. (2012). Beyond the colour of my skin: how skin colour affects the sense of body-ownership. Consciousness and Cognition, 21(3), 1242-1256.

Farmer, H., \& Tsakiris, M. (2012). The bodily social self: a link between phenomenal and narrative selfhood. Review of Philosophy and Psychology, 3(1), 125-144.

Làdavas, E. (2002). Functional and dynamic properties of visual peripersonal space. Trends in cognitive sciences, 6(1), 17-22.

Maister, L., Cardini, F., Zamariola, G., Serino, A., \& Tsakiris, M. (2015a). Your place or mine: Shared sensory experiences elicit a remapping of peripersonal space. Neuropsychologia, 70, 455-461.

Maister, L., Slater, M., Sanchez-Vives, M. V, \& Tsakiris, M. (2015b). Changing bodies changes minds: owning another body affects social cognition. Trends in Cognitive Sciences, 19(1), 6-12.

Serino, A., Pizzoferrato, F., \& Làdavas, E. (2008). Viewing a face (especially one's own face) being touched enhances tactile perception on the face. Psychological Science, 19(5), 434-438.

Tsakiris, M. (2008). Looking for myself: current multisensory input alters self-face recognition. PLoS One, 3(12), e4040. 
Tsakiris, M. (2010). My body in the brain: a neurocognitive model of body-ownership. Neuropsychologia, 48(3), 703-712. 